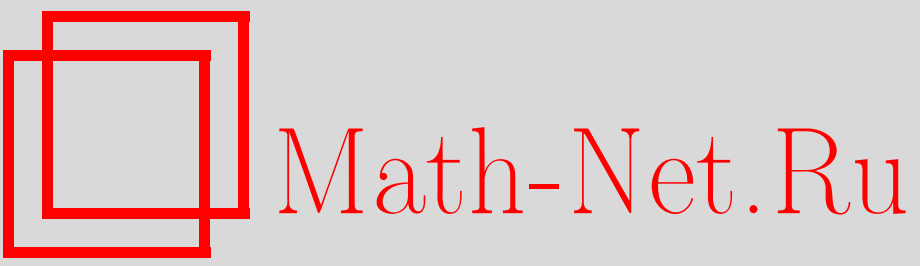

С. В. Козырев, А. Ю. Хренников, Псевдодифференциальные операторы на ультраметрических пространствах и ультраметрические всплески, Изв. РАН. Сер. матем., 2005, том 69, выпуск 5, 133-148

DOI: https://doi.org/10.4213/im657

Использование Общероссийского математического портала Math-Net.Ru подразумевает, что вы прочитали и согласны с пользовательским соглашением

http: //www . mathnet.ru/rus/agreement

Параметры загрузки:

IP: 54.174 .149 .18

26 апреля 2023 г., 16:39:11 


\title{
Псевдодифференциальные операторы на ультраметрических пространствах и ультраметрические всплески
}

\begin{abstract}
Строится анализ и спектральная теория псевдодифференциальных операторов и всплесков на ультраметрических пространствах общего вида. Вводятся операторы, обобщающие оператор Владимирова $p$-адического дробного дифференцирования. Строится семейство базисов ультраметрических всплесков в пространствах квадратично интегрируемых функций для широкого семейства ультраметрических пространств. Показано, что введенные всплески являются собственными векторами ультраметрических псевдодифференциальных операторов, и вычислены соответствующие собственные значения.

Библиография: 35 наименований.
\end{abstract}

\section{§1. Введение}

Настоящая работа посвящена исследованию спектральной теории псевдодифференциальных операторов и всплесков на ультраметрических пространствах. $p$-адические псевдодифференциальные операторы были рассмотрены в [1]-[7]. Важнейшим примером такого оператора является оператор Владимирова $p$-адического дробного дифференцирования, который может быть диагонализован $p$-адическим преобразованием Фурье. В настоящей работе мы вводим широкое семейство псевдодифференциальных операторов на более общих ультраметрических пространствах, которые не обязательно обладают структурой группы. Поскольку на общем ультраметрическом пространстве не существует преобразования Фурье, спектры введенных псевдодифференциальных операторов не могут быть исследованы при помощи этого преобразования. Вместо этого мы вводим и используем анализ ультраметрических всплесков на ультраметрических пространствах, строящихся как граница дерева.

В работах [8], [9] была введена конструкция Брюа-Титса деревьев, описываюших однородные пространства для некоторых групп $p$-адических матриц. В книге [10] рассматривались деревья и связанные с ними ультраметрические пространства. В работе [11] обсуждалась двойственность между ультраметрическими пространствами и направленньми деревьями. В работах [12], [13] исследовался

Работа первого автора была частично поддержана РФФИ (грант № 05-01-00884-а), грантом Президента Российской Федерации для поддержки научной школы (НШ 1542.2003.1), программой Отделения математики РАН "Современные проблемы теоретической математики” и грантом Шведской королевской академии наук для сотрудничества с учеными бывшего CCCP. 
класс ультраметрических пространств, представимых как граница дерева, и строились меры и ультраметрики на таких пространствах. В настоящей работе мы рассматриваем ультраметрическое пространство весьма общего вида, представимое как граница дерева, и, используя анализ на деревьях, вводим широкий класс псевдодифференциальных операторов на исследуемом ультраметрическом пространстве. Это показывает, что анализ ультраметрических псевдодифференциальных операторов связан с анализом на граффах.

В работе [14] был введен базис $p$-адических всплесков в пространстве $L^{2}\left(Q_{p}\right)$ квадратично интегрируемых комплекснозначных функций на поле $p$-адических чисел и было показано, что этот базис является базисом собственных векторов для оператора Владимирова. Была найдена связь этого базиса с обычным анализом всплесков на вешественной прямой. Из этого следует, что анализ всплесков (уже в вешественном случае) связан со спектральной теорией $p$-адических псевдодифференциальных операторов. Анализ всплесков есть хорошо развитый подход, используемый в широкой области приложений как в чистой, так и прикладной математике (см., например, обзор [15]). В работах [16], [17] были построены обобщения конструкции $p$-адических всплесков [14] для локально компактных абелевых групп.

В работе [18] было построено семейство псевдодифференциальных операторов в пространстве $L^{2}\left(Q_{p}\right)$, диагональных в базисе $p$-адических всплесков, но не диагонализуемых преобразованием Фурье, и вычислены соответствующие собственные значения. В настоящей работе мы обобшаем результаты работ [14] и [18] на случай широкого семейства ультраметрических пространств, соответствуюших направленным деревьям.

Мы вводим базисы ультраметрических всплесков на рассмотренном широком семействе ультраметрических пространств и доказываем, что базисы ультраметрических всплесков состоят из собственных векторов для введенных псевдодифференциальных операторов и вычисляем соответствующие собственные значения. Таким образом, для введенного класса ультраметрических пространств анализ всплесков позволяет исследовать спектры псевдодифференциальных операторов и, следовательно, является эффективной заменой анализа Фурье.

Тема настояшей работы связана с исследованиями, касаюшимися ультраметрической математической физики. Результаты $p$-адической математической физики см. в [1], [19]-[33]. Обзор применений метода реплик и ультраметричности в физике см. в [34], [35]. В [26], [27] было показано, что матрища Паризи, описьвающая нарушение репличной симметрии, есть дискретный аналог $p$-адического псевдодиффференциального оператора. В работах [28], [29] были рассмотрены матрицы Паризи, отвечающие более общим (чем поле $p$-адических чисел) абелевым локально компактным групам. В [33] обсуждалась связь ультраметрической дифффузии и динамики макромолекул. Другими применениями $p$-адической математической физики являются применения к математическим моделям биологии и науки о мышлении [22], [23].

В $\S 2$ настоящей работы мы строим семейство рассматриваемых ультраметрических пространств и вводим меры на этих пространствах. В $\S 3$ мы строим ортонормированные базисы ультраметрических всплесков в пространствах квадратично интегрируемых функций на ультраметрических пространствах, введенных в $\S 2$. 
В $\$ 4$ мы определяем псевдодифференциальные операторы, действующие на комплекснозначных функциях на пространствах, введенных в $\S 2$, доказываем, что эти операторы диагональны в базисах ультраметрических всплесков, и вычисляем соответствующие собственные значения. В $\S 5$ мы рассматриваем ультраметрическую замену переменной, отображаюшую рассматриваемые ультраметрические пространства на положительную полупрямую, и используем это отображение для построения неоднородного обобшения базисов всплесков.

\section{§2. Ультраметрические пространства $X(\mathcal{T})$}

В настоящем параграфе мы описьваем семейство ультраметрических пространств, связанных с деревьями.

Ультраметрическое пространство есть метрическое пространство с метрикой $d(x, y)$, удовлетворяюшей сильному неравенству треугольника

$$
d(x, y) \leqslant \max (d(x, z), d(y, z)) \quad \forall z .
$$

Точной верхней гранью $\sup S$ подмножества $S$ в частично упорядоченном множестве называется минимальньй элемент, больший или равньй всем элементам подмножества. Если любое конечное подмножество в частично упорядоченном множестве имеет единственную точную верхнюю грань, то частично упорядоченное множество называют направленным (а частичный порядок - направлением).

Напомним определения из теории графов.

Графр есть пара множеств $(G, E)$ (множество вершин и множество ребер), каждое из которых мы будем считать не более чем счетным. При этом каждое ребро $i \in E$ есть пара вершин $I_{0}, I_{1} \in G$ (начало и конец ребра), мы рассматриваем только такие ребра, для которых конец отличен от начала. В этом случае мы говорим, что $I_{0}, I_{1}$ соединены ребром, или являются соседними.

Путем в графе называем последовательность ребер такую, что конец предыдущего ребра совпадает с началом следующего. Длиной пути называется число ребер в этом пути. Граф̆ называется связным, если для любых двух вершин $I, J$ сушествует конечный путь с началом в $I$ и конщом в $J$. Расстоянием межсду вершинами графа называем длину соединяюшего их кратчайшего пути. Цикл в графе есть конечный путь, для которого начальная вершина совпадает с конечной. Дерево есть связный граф без циклов. Мы будем рассматривать только такие пути в деревьях, которые не имеют самопересечений.

Для описания ультраметрических пространств мы используем направленные деревья, т. е. деревья с частичным порядком, являющимся направлением.

Рассмотрим произвольное дерево $\mathcal{T}$ (конечное или бесконечное) такое, что количество ребер, входящих в каждую вершину, конечно. На дереве $\mathcal{T}$ мы определим направление (см. ниже), для которого любые две соседние вершины будут сравнимы. Индексом ветвления $p_{I}$ вершины $I$ в таком направленном дереве будем называть число убывающих ребер, выходящих из $I$ (ребро убывает, если его конец меньше начала).

Два пути в дереве $\mathcal{T}$ назовем әквивалентными, если они совпадают начиная с некоторой вершины (соответственно, они оба либо бесконечны, либо кончаются в одной вершине). Путь из класса эквивалентности $x$, начинающийся в вершине $A$, будем обозначать $A x$. 
Введем класс направлений на дереве $\mathcal{T}$.

ОПРЕДЕЛЕНИЕ 1. Фиксируем некий класс эквивалентности $\infty$ путей в дереве $\mathcal{T}$ (этот класс эквивалентности будем называть бесконечностью). Введем следуюший частичный порядок на дереве $\mathcal{T}: A<B$, где $A, B$ - вершины дерева $\mathcal{T}$, если сушествует путь из класса эквивалентности $\infty$ такой, что $A, B$ лежат на этом пути и $A<B$ в смысле порядка на пути (любой путь является вполне упорядоченным множеством в естественном смысле: вершина тем меньше, чем ближе к началу).

В этом случае $\infty$ - класс эквивалентности возрастающих путей в $\mathcal{T}$. Нетрудно проверить, что такой частичный порядок является направлением. Более того, любые соседние вершины дерева будут сравнимы.

Назовем бесконечно продолженным путь в направленном дереве $\mathcal{T}$ с описанным направлением, который либо бесконечен, либо заканчивается в вершине с индексом ветвления 0 , либо заканчивается в максимальной вершине в $\mathcal{T}$ (если такая вершина сушествует).

$A$ ссолютом $\widehat{X}(\mathcal{T})$ направленного дерева $\mathcal{T}$ будем называть множество классов эквивалентности бесконечно продолженных путей. Также рассмотрим множество $X(\mathcal{T})$ классов эквивалентности путей, содержащих убывающие бесконечно продолженные пути. Тогда абсолют $\widehat{X}(\mathcal{T})$ будет объединением $X(\mathcal{T}) \cup \infty$, т. е. $X(\mathcal{T})$ будет абсолютом с выколотой бесконечностью.

Множество $X(\mathcal{T})$ является ультраметрическим пространством (по отношению к естественно определенной метрике). Построим ультраметрику и меру на пространстве $X(\mathcal{T})$.

Для точек $x, y$ абсолюта существует единственный путь $x y$ в дереве $\mathcal{T}$. Здесь мы обозначаем через $x y$ следующее множество: в классе эквивалентности $x$ мы выбираем представитель $I x$ (путь к точке абсолюта $x$, начинающийся в вершине $I$ ), аналогично выбираем Iy и рассматриваем объединение $I x \cup I y$. Тогда мы определяем $x y$ как пересечение всех таких объединений:

$$
x y=\bigcap_{I}(I x \cup I y) .
$$

Множество ху мы будем называть путем между двумя точками абсолюта.

Для трех таких путей $x y, x z, y z$, отвечающих попарно различным точкам абсолюта $x, y, z$, сушествует единственная вершина $I$ дерева $\mathcal{T}$, лежащая на всех трех путях.

Точку пересечения трех путей $x y, x \propto, y \infty$ мы будем называть точной верхней гранью точек $x, y$ абсолюта и обозначать $\sup (x, y)$ :

$$
\sup (x, y)=x y \cap x \infty \cap y \infty .
$$

Аналогично, для вершин $I, J \in \mathcal{T}$

$$
\sup (I, J)=I J \cap I \infty \cap J \infty,
$$

где $I J$ - конечный путь, соединяющий $I$ и $J$, а также для $x \in X(\mathcal{T}), I \in \mathcal{T}$ введем

$$
\sup (I, x)=I x \cap I \infty \cap x \infty .
$$

Легко видеть, что определенная вьше $\sup (I, J)$ действительно будет точной верхней гранью в смысле определения 1.

$\Phi$ иксируем некоторую вершину $R$ дерева $\mathcal{T}$ (будем назьвать эту вершину корнем дерева). 
ОПРЕДЕЛЕНИЕ 2. Пусть $x, y \in X(\mathcal{T}), I=\sup (x, y)$ и $R$ - корень дерева. Расстоянием $d(x, y)$ между точками $X(\mathcal{T})$ является произведение индексов ветвления ребер вдоль конечного направленного пути $R I$ :

$$
R I=I_{0} \ldots I_{N}, \quad I_{0}=R, \quad I_{N}=I
$$

в степенях \pm 1 , причем индексы ветвления возрастаюших ребер $I_{j} I_{j+1}$ входят в произведение в степени +1 , а индексы ветвления убывающих ребер входят в степени -1 :

$$
d(x, y)=\prod_{j=0}^{N-1} p_{I_{j} I_{j+1}}^{\varepsilon_{I_{j} I_{j+1}}},
$$

где $\varepsilon_{I_{j} I_{j+1}}=1$ для $I_{j}<I_{j+1}, \varepsilon_{I_{j} I_{j+1}}=-1$ для $I_{j}>I_{j+1}$.

Если $x=y$, то расстояние $d(x, y)$ положим равным нулю.

Следующая теорема описывает семейство ультраметрик на направленных деревьях (мы называем две метрики на одном пространстве эквивалентны.ми, если они определяют одинаковые наборы шаров).

ТЕОРема 3. Если $F(I)$ - положительная возрастающая функиия на направленном дереве $\mathcal{T}$, то формула

$$
f(A, B)=F(\sup (A, B)), \quad A \neq B, \quad f(A, A)=0 \quad \forall A, B \in \mathcal{T},
$$

определяет на дереве $\mathcal{T}$ ультраметрику, т. е. әта функция неотрицательна, равна нулю только для $A=B$, симметрична по отношению $\kappa$ перестановке $A$ и $B$ и удовлетворяет сильному неравенству треугольника

$$
f(A, B) \leqslant \max (f(A, C), f(B, C)) \quad \forall A, B, C \in \mathcal{T}
$$

Более того, все ультраметрики, определенные таким образом, эквивалентны.

ДокАЗАТЕЛЬСтво. Для доказательства того, что $f(A, B)$ - ультраметрика, достаточно доказать, что $f(A, B)$ удовлетворяет сильному неравенству треугольника (остальные условия, необходимые для ультраметричности, очевидны).

Рассмотрим вершины $A, B, C$. Пусть $I=\sup (A, B), J=\sup (B, C), K=$ $\sup (A, C)$. Поскольку вершины $I$ и $K$ больше вершины $A$, то они сравнимы, иначе бы в дереве существовал цикл, являющийся объединением двух различных путей $A I \sup (I, K), A K \sup (I, K)$ (здесь $X Y Z$ обозначает путь, соединяюший вершины $X$ и $Z$ и проходящий через $Y$ ). Аналогично, сравнимы вершины $I$ и $J$, а также вершины $J$ и $K$. Следовательно, $\{I, J, K\}$ является вполне упорядоченным множеством.

Возможны два варианта: либо $I=J=K$, либо среди этих вершин существуют несовпадающие. Если $I=J=K$, то вследствие (3)

$$
f(A, B)=f(A, C)=f(B, C),
$$

т. е. вьполнено сильное неравенство треугольника. 
Пусть $I>J$, т. е. $\sup (A, B)>\sup (B, C)$. Тогда $\sup (A, C)=\sup (A, B)$, т. е. $I=K$. Это означает, что по формуле (3)

$$
f(A, B)=f(A, C)>f(B, C),
$$

т. е. вьполнено сильное неравенство треугольника.

Аналогично, при прочих выборах порядка на множестве $\{I, J, K\}$ мы опять получим сильное неравенство треугольника.

Поскольку структура множества шаров, определенная таким образом, зависит только от направления на дереве и не зависит от функции $F$, ультраметрики, соответствующие различным $F$, будут эквивалентны. Это завершает доказательство теоремы.

Так как пространство $X(\mathcal{T})$ есть множество классов эквивалентности бесконечно продолженных путей, совпадающих, начиная с некоторой вершины, с убывающими, то теорема 3 определяет на $X(\mathcal{T})$ ультраметрику, для которой

$$
f(x, y)=F(\sup (x, y))
$$

Поскольку функция (2) от вершины $I$, использованная в определении 2, является положительной возрастающей функцией, она определяет ультраметрику $d(x, y)$.

Мы определили ультраметрику на пространстве $X=X(\mathcal{T})$ (на абсолюте с выколотой бесконечностью). В топологии, отвечаюшей введенной ультраметрике, пространство $X$ будет локально компактно. Для дерева Брюа-Титса конструкция ультраметрики сводится в точности к определению $p$-адического расстояния.

Определим меру $\mu$ на пространстве $X(\mathcal{T})$, которая для случая дерева БрюаТитса сведется к мере Хаара на $p$-адических числах. Чтобы определить меру $\mu$, достаточно определить эту меру на шарах $D_{I}$, где $D_{I}$ - множество всех убывающих бесконечно продолженных путей, выходящих из вершины $I$. Напомним, что диаметр $d_{I}$ шара есть точная верхняя грань расстояния $d(x, y)$ между точками $x$ и $y$ в шаре $D_{I}$.

ОПРЕДЕЛЕНИЕ 4. Для шара $D_{I}$ ненулевого диаметра мера $\mu\left(D_{I}\right)$ равна его диаметру.

Для шара $D_{I}$ нулевого диаметра мера $\mu\left(D_{I}\right)$ определяется следуюшим образом: $\mu\left(D_{I}\right)=p_{I+1}^{-1}\left(D_{I+1}\right)$, где $I+1$ есть минимальная вершина, бо́льшая, чем $I$, $\mu\left(D_{I+1}\right)$ и $p_{I+1}$ суть мера соответствуюшего шара и индекс ветвления вершины $I+1$ соответственно.

Такую меру мы будем называть однородной. Поскольку шар $D_{I}$ содержит $p_{I}$ максимальных подшаров, которые по определению ультраметрики и меры имеют меру $p_{I}^{-1} \mu\left(D_{I}\right)$, то мера $\mu$ адлитивна на шарах. По адлитивности мы можем распространить меру на $\sigma$-алгебру борелевских множеств на пространстве $X$ ( $\sigma$-аддитивность меры $\mu$ будет следовать из локальной компактности $X$, аналогично случаю меры Лебега). Обозначим $L^{2}(X, \mu)$ пространство комплекснозначных квадратично интегрируемых (по отношению к введенной мере) функций на $X$. Поскольку $X$ (в общем случае) не является группой, не существует преобразования Фурье в $L^{2}(X, \mu)$. Тем не менее, в $L^{2}(X, \mu)$ можно ввести всплеск-преобразование. 
Обсудим представление элементов пространства $X(\mathcal{T})$ и направленного дерева $\mathcal{T}$, аналогичное разложению вещественных чисел в десятичные дроби (и $p$-адических чисел в ряды по степеням $p$ ). Естественно назвать введенную параметризацию иифровой параметризацией.

Для каждой вершины $I$ дерева $\mathcal{T}$ с индексом ветвления $p_{I}$ мы имеем $p_{I}$ убывающих ребер, выходящих из вершины, $0 \leqslant p_{I}<\infty$. Занумеруем все убывающие ребра числами $x_{I}=0, \ldots, p_{I}-1$ произвольным образом. Мы рассматриваем $x_{I}$ как функцию от $x \in D_{I}$, где $x_{I}$ - номер ребра, выходящего из вершины $I$, на убывающем пути из $I$ в $x$.

Рассмотрим точку $x \in X(\mathcal{T})$ и убываюший (бесконечный либо конечный) путь $\sup (R, x) x$, имеющий последовательность (убывающих) ребер $\left\{x_{L}\right\}$. Пусть $\gamma-$ число (возрастающих) ребер в конечном пути $R \sup (R, x)$.

Сопоставим точке $x$ последовательность номеров ребер (бесконечную для бесконечного пути и конечную для конечного пути)

$$
x_{-\gamma} x_{-\gamma+1} \ldots x_{-1}, x_{0} \ldots
$$

Элементы в приведенной последовательности есть элементы $\left\{x_{L}\right\}$, расположенные в порядке убывания.

Аналогично, вершине $I \in \mathcal{T}$ сопоставляется конечная последовательность $\left\{x_{L}\right\}$ номеров ребер в пути $\sup (R, I) I$ :

$$
x_{-\gamma} x_{-\gamma+1} \ldots x_{-1}, x_{0} \ldots x_{\beta},
$$

где $\gamma$ - число (возрастаюших) ребер в конечном пути $R \sup (R, I)$ и $x_{\beta}$ - номер убывающего ребра, входящего в $I$.

Легко видеть, что последовательности вида (4) являются пределами последовательностей вида (5), соответствуюших убываюшим вершинам, в норме (2). Данная конструкция есть полный аналог представления $p$-адического числа в виде ряда:

$$
x=\sum_{i=\gamma}^{\infty} x_{i} p^{i}, \quad x_{i}=0, \ldots, p-1
$$

(данный ряд - аналог последовательности (4)), и такие ряды являются пределами в $p$-адической норме конечных сумм вида

$$
x=\sum_{i=\gamma}^{\beta} x_{i} p^{i}, \quad x_{i}=0, \ldots, p-1
$$

(данный ряд - аналог последовательности (5)).

\section{§ 3. Базис всплесков в $L^{2}(X, \mu)$}

Для вершины $I$ дерева определим функцию $\chi_{I}(x)$ на пространстве $X$, равную характеристической функции шара $D_{I}$. Определим ультраметрический всплеск как функцию $\psi_{I j}(x)$ на $X$, где $I$ - вершина дерева и $j=1, \ldots, p_{I}-1$, заданную формулой

$$
\psi_{I j}(x)=\frac{e^{2 \pi i j x_{I} p_{I}^{-1}} \chi_{I}(x)}{\sqrt{\mu\left(D_{I}\right)}} .
$$


Определение всплеска зависит от нумерации ребер дерева (но носители всплесков не зависят от этой нумерации).

ТЕОРема 5. 1) Пусть в направленном дереве $\mathcal{T}$ существует бесконечная возрастающая последовательность шаров. Тогда набор функиий $\left\{\psi_{1 j}\right\}$, где I является элементом множества всех неминимальных вершин в дереве $\mathcal{T}$, есть ортонормированный базис в $L^{2}(X, \mu)$.

2) Пусть в направленном дереве $\mathcal{T}$ существует максимальная вершина $I_{f}$, для которой $\mu\left(D_{I_{f}}\right)=A$. Тогда набор функций $\left\{\psi_{I j}, A^{-\frac{1}{2}}\right\}$, где I является әлементом множества всех неминимальных вершин в дереве $\mathcal{T}$, есть ортонормированный базис в $L^{2}(X, \mu)$.

ДокАЗАТЕльство. Рассмотрим скалярное произведение

$$
\left\langle\psi_{I j}, \psi_{I^{\prime} j^{\prime}}\right\rangle=\frac{1}{\sqrt{\mu\left(D_{I}\right) \mu\left(D_{I^{\prime}}\right)}} \int e^{-2 \pi i j x_{I} p_{I}^{-1}} e^{2 \pi i j^{\prime} x_{I^{\prime}} p_{I^{\prime}}^{-1}} \chi_{I}(x) \chi_{I^{\prime}}(x) d \mu(x) .
$$

Поскольку для несравнимых $I, I^{\prime}$ носители соответствующих всплесков не пересекаются, то в этом случае скалярное произведение обращается в нуль.

Для сравнимых, но не совпадающих $I, I^{\prime}$, скалярное произведение (7) также обрашается в нуль, так как всплеск есть функция с нулевым средним, и для $I<I^{\prime}$ всплеск $\psi_{I^{\prime} j^{\prime}}$ есть константа на носителе всплеска $\psi_{I j}$.

Следовательно, скалярное произведение (7) может не быть равно нулю только для $I=I^{\prime}$, и мы получаем

$$
\left\langle\psi_{I j}, \psi_{I j^{\prime}}\right\rangle=\frac{1}{\mu\left(D_{I}\right)} \int e^{2 \pi i\left(j^{\prime}-j\right) x_{I} p_{I}^{-1}} \chi_{I}(x) d \mu(x)=\delta_{j j^{\prime}}
$$

Мы получили формулу

$$
\left\langle\psi_{I j}, \psi_{I^{\prime} j^{\prime}}\right\rangle=\delta_{I I^{\prime}} \delta_{j j^{\prime}}
$$

т. е. векторы $\psi_{I j}$ ортонормальны.

Поскольку множество индикаторов (характеристических функций) шаров $D_{I}$ по́лно в $L^{2}(X, \mu)$, то для доказательства полноты $\left\{\psi_{I j}\right\}$ достаточно доказать равенство Парсеваля для индикаторов $\chi_{J}(x)$.

Для нормированных индикаторов имеет место следующее скалярное произведение:

$$
\frac{1}{\sqrt{\mu\left(D_{J}\right)}}\left\langle\chi_{J}, \psi_{I j}\right\rangle=\frac{1}{\sqrt{\mu\left(D_{I}\right) \mu\left(D_{J}\right)}}\left\langle\chi_{J}(x), e^{2 \pi i j x_{I} p_{I}^{-1}} \chi_{I}(x)\right\rangle
$$

равное

$$
\sqrt{\frac{\mu\left(D_{J}\right)}{\mu\left(D_{I}\right)}} e^{2 \pi i j x_{I} p_{I}^{-1}}
$$

для $J<I$ и равное нулю в противном случае. Отсюда следует тождество

$$
\sum_{I, j}\left|\frac{1}{\sqrt{\mu\left(D_{J}\right)}}\left\langle\chi_{J}, \psi_{I j}\right\rangle\right|^{2}=\mu\left(D_{J}\right) \sum_{I>J, j} \frac{1}{\mu\left(D_{I}\right)}=\mu\left(D_{J}\right) \sum_{I>J} \frac{p_{I}-1}{\mu\left(D_{I}\right)}
$$


где суммирование ведется по возрастающему пути в $\mathcal{T}$, начинающемуся в вершине $J$.

Рассмотрим возрастающую последовательность вершин $J \infty: \quad J=I_{0}<$ $I_{1}<\ldots$, начинающихся с $J$ (отметим, что эта последовательность может быть конечной, когда $\infty$ отождествляется с максимальной вершиной $I_{f}$ в $\mathcal{T}$ ). Для вершин из этой последовательности рассмотрим тождество

$$
\mu\left(D_{I_{k}}\right)=\mu\left(D_{J}\right) \prod_{l=1}^{k} p_{I_{l}} .
$$

Отсюда формула (9) принимает следуюший вид:

$$
\begin{aligned}
\mu\left(D_{J}\right) \sum_{I>J} \frac{p_{I}-1}{\mu\left(D_{I}\right)} & =\sum_{k=1}^{f} \frac{p_{I_{k}}-1}{\prod_{l=1}^{k} p_{I_{l}}} \\
& =\sum_{k=1}^{f}\left[\left(\prod_{l=1}^{k-1} p_{I_{l}}\right)^{-1}-\left(\prod_{l=1}^{k} p_{I_{l}}\right)^{-1}\right]=1-\left(\prod_{l=1}^{f} p_{I_{l}}\right)^{-1}
\end{aligned}
$$

когда в последовательности $J \propto$ сушествует максимальная вершина $I_{f}$, и принимает вид

$$
\lim _{f \rightarrow \infty}\left[1-\left(\prod_{l=1}^{f} p_{I_{l}}\right)^{-1}\right]=1,
$$

когда такой вершины не существует. Это доказывает утверждение 1) теоремы.

Поскольку добавка в правой части (10) соответствует вкладу от соответствующим образом нормированной константы (когда в $\mathcal{T}$ существует максимальная вершина и объем $X(\mathcal{T})$ конечен), то мы получаем доказательство утверждения 2) теоремы.

Введенный в теореме 5 базис мы будем называть базисом ультраметрических всплесков (соответствуюшим образом нормированную константу мы также будем называть всплеском). Для $p$-адического случая этот базис сводится к базису $p$-адических всплесков, введенных в [14].

\section{§4. Псевдодифференциальные операторы}

В настоящем параграфе мы изучаем ультраметрический псевдодифференщиальный оператор (или ПДО) вида

$$
T f(x)=\int T(x, y)(f(x)-f(y)) d \mu(y)
$$

где ядро интегрирования $T(x, y)$ симметрично и локально постоянно по $y$ при фиксированном $x$ (вне любой окрестности $x$ ) и для произвольного фиксированного $x$ вьполнено следующее условие:

$$
T(x, y)=\text { const, } \text { если } d(x, y)=\text { const. }
$$


Теорема 6. Пусть $T(I)$ - комплекснозначная функиия на направленном дереве $\mathcal{T}$. Тогда функиия

$$
T(x, y)=T(\sup (x, y))
$$

симметрична и локально постоянна по у при фиксированном $x$ (вне любой окрестности $x$ ) и для произвольного фиксированного х выполнено следующее условие:

$$
T(x, y)=\text { const }, \quad e c \bumpeq u \quad d(x, y)=\text { const } .
$$

Обратно, для любой такой функции $T(x, y)$ существует такая функиия $T(I)$ на направленном дереве $\mathcal{T}$, что функиия $T(x, y)$ определяется формулой (12).

Это соответствие задается формулой

$$
T(x, y)=T(\sup (x, y))=\sum_{I} T(I) \delta_{\operatorname{diam}\left(D_{I}\right), d(x, y)} \chi_{I}(x) .
$$

Здесь $\operatorname{diam}\left(D_{I}\right)-$ диаметр иара $D_{I}$.

ДокАЗАТЕЛЬСТво. Очевидно, что функция (12) удовлетворяет условию (13). Обратно, рассматривая функцию $T(x, y)$, удовлетворяющую $(13)$, и определяя $T(I)$ как значение $T(x, y)$ для $x \in D_{I}, y \in D_{I}, d(x, y)=\operatorname{diam}\left(D_{I}\right)$, получим (12). Формула (14) дает описание такой процедуры. Это завершает доказательство теоремы.

ТЕОРема 7. Пусть абсолютно сходится следующий ряд:

$$
\sum_{J>R} T(J) \mu\left(D_{J}\right)<\infty
$$

Тогда оператор (11) является плотно определенным оператором в $L^{2}(X, \mu)$ и всплески $\psi_{\text {I }}$ являются его собственными векторами:

$$
T \psi_{I j}(x)=\lambda_{I} \psi_{I j}(x)
$$

с собственными значениями

$$
\lambda_{I}=T(I) \mu\left(D_{I}\right)+\sum_{J>I} T(J) \mu\left(D_{J}\right)\left(1-p_{J}^{-1}\right) .
$$

Oператор T самосопряжен, если функция $T(I)$ вещественнозначна, и положителен, если эта функция неотрицательна.

Доказательство. Рассмотрим всплеск $\psi_{I j}$. Тогда

$$
T \psi_{I j}(x)=\int T(x, y)\left(\psi_{I j}(x)-\psi_{I j}(y)\right) d \mu(y)
$$


Рассмотрим следующие случаи.

1) Пусть $x$ лежит вне $D_{I}$. Тогда

$$
T \psi_{I j}(x)=-T(\sup (x, I)) \int \psi_{I j}(y) d \mu(y)=0
$$

2) Пусть $x \in D_{I}$. Тогда

$$
\begin{aligned}
T \psi_{I j}(x) & =\left(\int_{d(x, y)>\operatorname{diam}\left(D_{I}\right)}+\int_{d(x, y)=\operatorname{diam}\left(D_{I}\right)}\right) T(x, y)\left(\psi_{I j}(x)-\psi_{I j}(y)\right) d \mu(y) \\
& \left.+\int_{d(x, y)<\operatorname{diam}\left(D_{I}\right)}\right) T(x, y)\left(\psi_{I j}(x)-\psi_{I j}(y)\right) d \mu(y) \\
= & \left(\int_{d(x, y)>\operatorname{diam}\left(D_{I}\right)}+\int_{d(x, y)=\operatorname{diam}\left(D_{I}\right)} T(x, y) d \mu(y)\right. \\
= & \psi_{I j}(x) \int_{d(x, y)>\operatorname{diam}\left(D_{I}\right)} T(x, y)\left(\psi_{I j}(x)-\psi_{I j}(y)\right) d \mu(y) \\
& +\int_{d(x, y)=\operatorname{diam}\left(D_{I}\right)} T(\sup (I, y)) d \mu(y) \\
= & \psi_{I j}(x) \int_{\sup (I, y)>I} p_{I-1}\left(1-e^{2 \pi i j l}\right) . \\
& +T(I) \psi_{I j}(x) \mu\left(D_{I}\right) p_{I}^{-1} \sum_{l=1}
\end{aligned}
$$

Последнее равенство следует из локального постоянства $\psi_{I j}$.

Поскольку при $j=1, \ldots, p-1 \bmod p$ имеет место равенство

$$
\sum_{l=1}^{p-1}\left(1-e^{2 \pi i p^{-1} j l}\right)=p
$$

получаем

$$
T \psi_{I j}(x)=\psi_{I j}(x)\left(\int_{\sup (I, y)>I} T(\sup (I, y)) d \mu(y)+T(I) \mu\left(D_{I}\right)\right) .
$$

Вычислим

$\int_{\sup (I, y)>I} T(\sup (I, y)) d \mu(y)+T(I) \mu\left(D_{I}\right)=T(I) \mu\left(D_{I}\right)+\sum_{J>I} T(J) \mu\left(D_{J}\right)\left(1-p_{J}^{-1}\right)$,

если соответствующий ряд сходится. Поскольку все растущие пути в дереве $\mathcal{T}$ совпадают начиная с некоторой вершины, то сходимость всех таких рядов обеспечивается абсолютной сходимостью ряда (15). Это завершает доказательство теоремы. 


\section{§5. Связь со всплесками на вещественной прямой}

В настоящем параграфе мы рассматриваем случай, когда в дереве $\mathcal{T}$ все возрастающие и убывающие пути бесконечны.

В работе [14] обсуждалась связь между базисом $\left\{\Psi_{\gamma j n}\right\}$ p-адических всплесков и базисом всплесков в пространстве квадратично интегрируемых функций $L^{2}\left(\mathbb{R}_{+}\right)$ на положительной вешественной полупрямой.

Рассматривалась следующая $p$-адическая замена переменной:

$$
\begin{gathered}
\eta: Q_{p} \rightarrow \mathbb{R}_{+}, \\
\eta: \sum_{i=\gamma}^{\infty} a_{i} p^{i} \mapsto \sum_{i=\gamma}^{\infty} a_{i} p^{-i-1}, \quad a_{i}=0, \ldots, p-1, \quad \gamma \in \mathbb{Z},
\end{gathered}
$$

отображающая базис всплесков на базис $p$-адических всплесков.

$p$-адическая замена переменной, примененная к базису $p$-адических всплесков, породит базис в $L^{2}\left(\mathbb{R}_{+}\right)$, векторы из которого с точностью до умножения на число имеют вид

$$
\Psi_{\gamma n_{j}}^{(p)}(x)=p^{-\frac{\gamma}{2}} \Psi_{j}^{(p)}\left(p^{-\gamma} x-n\right), \quad \gamma \in \mathbb{Z}, \quad n \in \mathbb{Z}_{+},
$$

где $\mathbb{Z}_{+}-$множество положительных целых чисел и

$$
\Psi_{j}^{(p)}(x)=\sum_{l=0}^{p-1} e^{2 \pi i j p^{-1}} \chi_{\left[l p^{-1},(l+1) p^{-1}\right]}(x) .
$$

Такой базис есть обобшение базиса всплесков, порожденного всплеском Хаара (и расширяется до базиса в $L^{2}\left(\mathbb{R}_{+}\right)$, если $n \in \mathbb{Z}_{+}$). Для $p=2$ мы получим базис всплесков Хаара.

Построенньй в настоящей работе базис $\left\{\psi_{I j}\right\}$ порождает новый базис в $L^{2}\left(\mathbb{R}_{+}\right)$, являюшийся обобщением базиса всплесков.

В настоящей работе мы строим обобщение отображения $\eta$, которое мы будем называть ультраметрической заменой переменной и обозначать $\rho: X \rightarrow \mathbb{R}_{+}$. Для точки $x$ пространства $X(\mathcal{T})$ :

$$
x=x_{I_{\gamma}} x_{I_{\gamma+1}} \ldots x_{I_{-1}}, x_{I_{0}} x_{I_{1}} \ldots, \quad x_{I}=0, \ldots, p_{I}-1, \quad \gamma \in \mathbb{Z},
$$

отображение $\rho$ имеет вид

$$
\rho: x \mapsto \sum_{k=\gamma}^{-1} x_{I_{k}} \prod_{l=k}^{-1} p_{I_{l}}+\sum_{k=0}^{\infty} x_{I_{k}} \prod_{l=0}^{k} p_{I_{l}}^{-1}
$$

для отрицательных $\gamma$ и вид

$$
\rho: x \mapsto \sum_{k=\gamma}^{\infty} x_{I_{k}} \prod_{l=0}^{k} p_{I_{l}}^{-1}
$$

для положительных $\gamma$.

Это отображение не является взаимно однозначным (но является взаимно однозначным почти всюду). Отображение $\rho$ непрерьвно, и можно доказать следующую лемму. 
Лемма 8. Отображение $\rho$ удовлетворяет неравенству Гёльдера

$$
|\rho(x)-\rho(y)| \leqslant d(x, y)
$$

ДоказАтельСтво. Рассмотрим

$$
x=x_{I_{\alpha}} x_{I_{\alpha+1}} \ldots x_{I_{-1}}, x_{I_{0}} x_{I_{1}} \ldots, \quad y=y_{J_{\beta}} y_{J_{\beta+1}} \ldots y_{J_{-1}}, y_{J_{0}} y_{J_{1}} \ldots,
$$

где мы принимаем без потери обшности, что $\alpha \leqslant \beta$. Для простоты предположим $0 \leqslant \alpha \leqslant \beta$. В этом случае

$$
\begin{array}{ll}
x=0,0 \ldots 0 x_{I_{\alpha}} \ldots, & \rho(x)=\sum_{k=\alpha}^{\infty} x_{I_{k}} \prod_{l=0}^{k} p_{I_{l}}^{-1}, \\
y=0,0 \ldots 0 y_{I_{\beta}} \ldots, & \rho(y)=\sum_{k=\beta}^{\infty} y_{J_{k}} \prod_{l=0}^{k} p_{J_{l}}^{-1} .
\end{array}
$$

Тогда

$$
d(x, y)=\prod_{l=0}^{\alpha-1} p_{I_{l}}^{-1}
$$

Имеют место соотношения

$$
\begin{aligned}
\rho(x)-\rho(y) & =\sum_{k=\alpha}^{\beta-1} x_{I_{k}} \prod_{l=0}^{k} p_{I_{l}}^{-1}+\sum_{k=\beta}^{\infty}\left[x_{I_{k}} \prod_{l=0}^{k} p_{I_{l}}^{-1}-y_{J_{k}} \prod_{l=0}^{k} p_{J_{l}}^{-1}\right] \\
& =d(x, y)\left(\sum_{k=\alpha}^{\beta-1} x_{I_{k}} \prod_{l=\alpha}^{k} p_{I_{l}}^{-1}+\sum_{k=\beta}^{\infty}\left[x_{I_{k}} \prod_{l=\alpha}^{k} p_{I_{l}}^{-1}-y_{J_{k}} \prod_{l=\alpha}^{k} p_{J_{l}}^{-1}\right]\right) \\
& \leqslant d(x, y)\left(\sum_{k=\alpha}^{\beta-1}\left(p_{I_{k}}-1\right) \prod_{l=\alpha}^{k} p_{I_{l}}^{-1}+\sum_{k=\beta}^{\infty}\left(p_{I_{k}}-1\right) \prod_{l=\alpha}^{k} p_{I_{l}}^{-1}\right) \\
& =d(x, y) \sum_{k=\alpha}^{\infty}\left(p_{I_{k}}-1\right) \prod_{l=\alpha}^{k} p_{I_{l}}^{-1}=d(x, y) \lim _{f \rightarrow \infty}\left(1-\prod_{l=\alpha}^{f} p_{I_{l}}^{-1}\right)=d(x, y),
\end{aligned}
$$

что завершает доказательство леммы.

Лемма 9. Отображсение $\rho$ удовлетворяет условиям

$$
\begin{aligned}
\rho: D_{I} & \rightarrow \rho(I)+\left[0, \mu\left(D_{I}\right)\right], \\
\rho: X \backslash D_{I} & \rightarrow \mathbb{R}_{+} \backslash\left\{\rho(I)+\left[0, \mu\left(D_{I}\right)\right]\right\}
\end{aligned}
$$

с точностью до конечного числа точек.

Отметим, что здесь мы отождествляем вершину $I$ и точку $X$ с нумерацией $I 00 \ldots$. 
ДокАЗАТЕЛЬСТво. Для вершины $I$ рассмотрим точки

$$
\tilde{I}=x_{I_{\alpha}} x_{I_{\alpha+1}} \ldots x_{I_{-1}}, x_{I_{0}} x_{I_{1}} \ldots x_{I_{\beta-1}} 0 \ldots
$$

и

$$
\tilde{\tilde{I}}=x_{I_{\alpha}} x_{I_{\alpha+1}} \ldots x_{I_{-1}}, x_{I_{0}} x_{I_{1}} \ldots x_{I_{\beta-1}} p_{I_{\beta}}-1, \ldots
$$

Точка $\tilde{I}$ является точкой пространства $X$, отвечающей вершине $I$. Тогда

$$
\begin{aligned}
& \rho(\tilde{I})=\sum_{k=\alpha}^{-1} x_{I_{k}} \prod_{l=k}^{-1} p_{I_{l}}+\sum_{k=0}^{\beta-1} x_{I_{k}} \prod_{l=0}^{k} p_{I_{l}}^{-1}, \\
& \rho(\tilde{\tilde{I}})=\sum_{k=\alpha}^{-1} x_{I_{k}} \prod_{l=k}^{-1} p_{I_{l}}+\sum_{k=0}^{\beta-1} x_{I_{k}} \prod_{l=0}^{k} p_{I_{l}}^{-1}+\sum_{k=\beta}^{\infty}\left(p_{I_{k}}-1\right) \prod_{l=0}^{k} p_{I_{l}}^{-1} .
\end{aligned}
$$

Имеем

$$
\begin{aligned}
\rho(\tilde{\tilde{I}})-\rho(\tilde{I}) & =\sum_{k=\beta}^{\infty}\left(p_{I_{k}}-1\right) \prod_{l=0}^{k} p_{I_{l}}^{-1}=\prod_{l=0}^{\beta-1} p_{I_{l}}^{-1} \sum_{k=\beta}^{\infty}\left(p_{I_{k}}-1\right) \prod_{l=\beta}^{k} p_{I_{l}}^{-1} \\
& =\mu\left(D_{I}\right) \lim _{f \rightarrow \infty}\left(1-\prod_{l=\beta}^{f} p_{I_{l}}^{-1}\right)=\mu\left(D_{I}\right) .
\end{aligned}
$$

Тогда

$$
\rho(\tilde{\tilde{I}})=\rho(\tilde{I})+\mu\left(D_{I}\right) .
$$

Используя лемму 8, мы получим доказательство настоящей леммы.

ЛЕмма 10. Отображение $\rho$ переводит меру $\mu$ на пространстве $X$ в меру Лебега $l$ на $\mathbb{R}_{+}:$для любого измеримого подмножества $S \subset X$ выполнено равенство

$$
\mu(S)=l(\rho(S)),
$$

или, в символьных обозначениях,

$$
\rho: d \mu(x) \mapsto d x .
$$

ДокАЗАТЕЛЬСтво. Из леммы 9 следует, что шары в $X$ отображаются на замкнутые промежутки в $\mathbb{R}_{+}$с сохранением меры. Отображение $\rho: X \rightarrow \mathbb{R}_{+}$сюръективно, и, поскольку непересекающиеся шары отображаются на промежутки, которые не пересекаются или имеют пересечение меры нуль (по лемме 9), это доказывает настоящую лемму.

Следовательно, соответствующее сопряженное отображение

$$
\rho^{*}: L^{2}\left(\mathbb{R}_{+}\right) \rightarrow L^{2}(X), \quad \rho^{*} f(x)=f(\rho(x)),
$$

есть унитарный оператор. Обратное к этому отображение будет переводить базис ультраметрических всплесков на пространстве $X$ в некоторый базис из функций на положительной вещественной полуоси. 
Отображение $\rho^{-1 *}$ между пространствами квадратично интегрируемых фуункций хорошо определено, поскольку отображение $\rho$ взаимно однозначно на множестве полной меры.

Используя ультраметрическую замену переменной $\rho$, мы можем определить новые примеры всплесков в $L^{2}\left(\mathbb{R}_{+}\right)$. Назовем такие всплески неоднороднылми, поскольку базис $\rho^{-1 *}\left\{\psi_{I j}\right\}$ в $L^{2}\left(\mathbb{R}_{+}\right)$не имеет транслящионной инвариантности (сдвиг всплеска не обязательно является всплеском, в то время как для обычных кратномасштабных всплесков это было бы так).

ОПРЕДЕЛЕНИЕ 11 . Базис $\left\{\Psi_{I j}\right\}$ в $L^{2}\left(\mathbb{R}_{+}\right)$, где $\Psi_{I j}=\rho^{-1 *} \psi_{I j}$, называется базисом неоднородных всплесков на положительной вещественной полупрямой.

Базис неоднородных всплесков объединяет всплески, соответствующие $p$-адическим всплескам для разных $p$.

Авторы благодарны И.В. Воловичу, В.С. Владимирову, Дж. Паризи и В. А. Аветисову за плодотворные обсуждения и важные замечания. Один из авторов (А. Хренников) благодарен С. Альбеверио за плодотворные обсуждения и поддержку $p$-адических исследований. Настоящая работа была частично поддержана сетью Европейского сообщества по квантовой вероятности и применениям.

\section{Список литературы}

1. Владимиров В.С., Волович И. В., Зеленов Е.И. p-адический анализ и математическая физика. М.: Наука, 1994.

2. Владимиров В. С. О спектре некоторых псевдодифференциальных операторов над полем $p$-адических чисел // Алгебра и анализ. 1990. Т. 2. №6. С. 107-124.

3. Владимиров В. С. О спектральных свойствах $p$-адических псевдодифференциальных операторов типа Шрёдингера // Изв. РАН. Сер. матем. 1992. Т. 56. № 4. С. 770-789.

4. Хренников А. Ю. Фундаментальные решения над полем $p$-адических чисел // Алгебра и анализ. 1992. Т. 4. № 3. С. 248-266.

5. Albeverio S., Karwowosky W. A random walk on $p$-adic numbers // Stochastic Process-Physics and Geometry II / Eds. S. Albeverio, U. Cattaneo, D. Merlini. Singapore: World Scientific, 1995. P. 61-74.

6. Kochubei A.N. Pseudo-Differential Equations and Stochastics over Non-Archimedean Fields. N. Y.: Marcel Dekker, 2001.

7. Кочубей A. Н. Фундаментальные решения псевдодифференциальных уравнений, связанных с $p$-адическими квадратичными формами // Изв. РАН. Сер. матем. 1998. Т. 62 . № 6. C. $103-124$.

8. Bruhat F., Tits J. Groupes reductifs sur un corps local // Inst. Hautes Etudes Sci. Publ. Math. 1972. V. 41. P. 5-251.

9. Bruhat F., Tits J. Groupes reductifs sur un corps local. II. Schemas en groupes. Existence d'une donnee radicielle valuee // Inst. Hautes Etudes Sci. Publ. Math. 1984. V. 60. P. $197-376$.

10. Serre J.P. Trees. N.Y. -Berlin: Springer-Verlag, 1980.

11. Lemin A. J. The category of ultrametric spaces is isomorphic to the category of complete, atomic, tree-like and real graduated lattices LAT // Algebra universalis. 2003. V. 50. № 1. P. 35-49.

12. Cohen J. M., Colonna F., Singman D. Distributions and measures on the boundary of a tree // J. Math. Anal. Appl. 2004. V. 293. P. 89-107.

13. Choucroun F. Arbres, espaces ultrametriques et bases de structure uniforme // Geom. Dedicata. 1994. V. 53. P. 69-74. 
14. Козырев C. В. Анализ всплесков как $p$-адический спектральный анализ // Изв. РАН. Cер. матем. 2002. Т. 66. № 2. С. 149-158.

15. Daubechies I. Ten Lectures on Wavelets // CBMS Lect. Not. Ser. Philadelphia: SIAM, 1991.

16. Benedetto J.J., Benedetto R.L. A wavelet theory for local fields and related groups // J. Geom. Anal. 2004. V. 14. № 3. P. 423-456.

17. Benedetto R.L. Examples of wavelets for local fields. http://arxiv.org/math.CA/abs /0312038.

18. Козырев С. В. $p$-адические псевдодифференциальные операторы и $p$-адические всплески // ТМФ. 2004. Т. 138. № 3. С. 383-394.

19. Volovich I. V. p-Adic String // Class. Quantum Gravity. 1987. V. 4. P. L83-L87.

20. Aref'eva I. Ya., Dragovic B., Frampton P., Volovich I. V. Wave function of the universe and $p$-adic gravity // Mod. Phys. Lett. A. 1991. V. 6. P. 4341-4358.

21. Khrennikov A. p-Adic valued distributions in mathematical physics. Dordrecht: Kluwer Academic Publ., 1994.

22. Khrennikov A. Non-Archimedean Analysis: Quantum Paradoxes, Dynamical Systems and Biological Models. Dordrecht: Kluwer Academic Publ., 1997.

23. Khrennikov A. Classical and quantum mental models and Freud's theory of unconscious mind. Växjö: Växjö University press, 2002.

24. Хренников А. Ю. Неархимедов анализ и его приложения. М.: Наука, 2003.

25. Khrennikov A. p-Adic discrete dynamical systems and their applications in physics and cognitive sciences // Russian J. Math. Phys. 2004. V. 11. № 1. P. 45-70.

26. Avetisov V.A., Bikulov A.H., Kozyrev S. V. Application of $p$-adic analysis to models of spontaneous breaking of the replica symmetry // J. Phys. A: Math. Gen. 1999. V. 32. P. $8785-8791$.

27. Parisi G., Sourlas N. p-Adic numbers and replica symmetry breaking // European Phys. J. B. 2000. V. 14. P. 535-542.

28. Carlucci D. M., De Dominicis C. On the replica Fourier transform // Comptes Rendus Ac. Sci. Ser. IIB. Mech. Phys. Chem. Astr. 1997. V. 325. P. 527.

29. De Dominicis C., Carlucci D. M., Temesvari T. Replica Fourier transform on ultrametric trees and block-diagonalizing multireplica matrices // J. Phys. I France. 1997. V. 7. P. $105-115$.

30. Ogielski A.T., Stein D.L. Dynamics on ultrametric spaces // Phys. Rev. Lett. 1985. V. 55. № 15. P. 1634-1637.

31. Schreckenberg M. Long run diffusion on ultrametric spaces // Zeitschrift für Physik B Condensed Matter. 1985. V. 60. P. 483-488.

32. Huberman B. A., Kerszberg M. Ultradiffusion: the relaxation on hierarchical systems // J. Phys. A: Math. Gen. 1985. V. 18. P. L331-L336.

33. Avetisov V.A., Bikulov A.H., Kozyrev S. V., Osipov V. A. p-Adic Models of Ultrametric Diffusion Constrained by Hierarchical Energy Landscapes // J. Phys. A: Math. Gen. 2002. V. 35. P. 177-189.

34. Mezard M., Parisi G., Virasoro M. Spin-Glass Theory and Beyond. Singapore: World Scientific, 1987.

35. Rammal R., Toulouse G., Virasoro M.A. Ultrametricity for physicists // Rev. Mod. Phys. 1986. V. 58. P. 765-788. 